\title{
Minimally Invasive Transanal Repair of Rectourethral Fistulas
}

\author{
Giulio Nicita *, Donata Villari, Simone Caroassai Grisanti, Michele Marzocco, \\ Vincenzo Li Marzi, Alberto Martini
}

Department of Urology, University of Florence, Careggi Hospital, Florence, Italy

\section{Article info}

\section{Article history:}

Accepted June 6, 2016

Associate Editor:

Alexandre Mottrie

\section{Keywords:}

Fistula treatment

Laparoscopic instruments

Minimally invasive surgery

Transanal approach

\section{Please visit \\ www.europeanurology.com and www.urosource.com to view the accompanying video.}

\begin{abstract}
Background: Rectourethral fistulas (RUFs) represent an uncommon complication of pelvic surgery, especially radical prostatectomy. To date there is no standardised treatment for managing RUFs. This represents a challenge for surgeons, mainly because of the potential recurrence risk.

Objective: To describe our minimally invasive transanal repair (MITAR) of RUFs and to assess its safety and outcomes.

Design, setting, and participants: We retrospectively evaluated 12 patients who underwent MITAR of RUF at our centre from October 2008 to December 2014. Exclusion criteria were a fistula diameter greater than $1.5 \mathrm{~cm}$, sepsis, and/or faecaluria.

Surgical procedure: After fistula identification through cystoscopy and 5F-catheter positioning within the fistula, MITAR is performed using laparoscopic instruments introduced through Parks' anal retractor. The fibrotic margins of the fistula are carefully dissected by a lozenge incision of the rectal wall, parallel to the rectal axis. Under the healthy flap of the rectal wall the urothelium is located and the fistulous tract is sutured with interrupted stitches. After a leakage test of the bladder, the rectal wall is sutured with interrupted stitches. Electrocoagulation is never used during this procedure.

Measurements: Fistula closure, postoperative complications, and recurrence.

Results and limitations: Median follow-up was 21 (range, 12-74) mo. Median operative time was 58 (range, 50-70) min. Median hospital stay was 1.5 (range, 1-4) d. Early surgical complications occurred in one patient (8.3\%). Recurrence did not occur in any of the cases. Limitations included retrospective analysis, small case load, and lack of experience with radiation-induced fustulas.

Conclusions: MITAR is a safe, effective, and reproducible procedure. Its advantages are low morbidity and quick recovery, and no need for a colostomy.

Patient summary: We studied the treatment of rectourethral fistulas. Our technique, transanally performed using laparoscopic instruments, was found to be safe, feasible, and effective, with limited risk of complications.

(C) 2016 Published by Elsevier B.V. on behalf of European Association of Urology.

\footnotetext{
* Corresponding author. Clinica Urologica II, Azienda Ospedaliera Careggi, Università di Firenze, Viale San Luca, Firenze 50134, Italy. Tel. +390557949218; fax: +390552758014.

E-mail address: gnicita@unifi.it (G. Nicita).
}

\section{Introduction}

Rectourethral fistulas (RUFs) are tracts that pathologically connect the rectum to the urethra. They usually represent a rare but significant complication of radical prostatectomy and one that is difficult to resolve [1]. RUFs are usually located at or near the vesico-urethral anastomosis and the membranous urethra [2]. RUF incidence varies from $0.6 \%$ to $9 \%[3,4]$. Intraoperative accidental rectal injury is a major risk factor for the formation of a fistula [5]. 
With the improvements in multimodal prostate cancer (PCa) treatments, the incidence of fistulas induced by brachytherapy, cryotherapy, or radiotherapy (RT) has risen [6]. These fistulas are usually large $(>1.5 \mathrm{~cm})$ and difficult to treat because they present thick, fibrotic hypovascular surrounding tissue [7].

Several techniques can be used for managing RUFs: transanal, transabdominal, trans-sphincteric, and transperineal; the last being most commonly adopted [6]. To date no standardised RUF treatment exists. In fact, the majority of published studies concern single institution experiences, small case series, or single case reports. RUF treatment represents a challenge for surgeons, especially because of the risk of potential recurrence.

The aim of this paper is to report our experience with a minimally invasive transanal repair (MITAR) technique performed for treating fistulas smaller than $1.5 \mathrm{~cm}$ using laparoscopic instruments, and no need for colostomy. Our procedure precludes the interposition of a tissue flap between the rectum and the urothelium after their repair. For this reason our technique is not appropriate for treating fistulas at a high risk of recurrence in which case it is advisable to separate the sutures by a buffer tissue [8].

\section{Patients and methods}

\subsection{Study population and design}

From October 2008 to December 2014, 12 patients (1.2\%) out of a total of 983 RPs (324 open and 659 robotic) developed RUF suitable for MITAR. Exclusion criteria for minimally invasive repair were fistula diameter greater than $1.5 \mathrm{~cm}$, sepsis, and/or faecaluria. We arbitrarily set the cutoff value at $1.5 \mathrm{~cm}$ because this already represents a wide fistula and it becomes wider after fibrotic margin resection.

Baseline patient characteristics are listed in Table 1. Open radical retropubic prostatectomy was performed in four patients, while roboticassisted radical retropubic prostatectomy was performed in eight. In four patients, out of eight intraoperatively detected rectal injuries, the fistula resulted from a failure of a two-layer suture.

Median age at MITAR was $63 \mathrm{yr}$ (range, 54-71). Clinical suspicion was aroused by pneumaturia, cloudy urine, and urinary rectal discharge. These appeared after a median of $13 \mathrm{~d}$ (range, 2-26). No patient presented copious faecaluria at diagnosis. None had either diabetes mellitus or inflammatory bowel disease. One patient had undergone bowel surgery for sigmoid diverticula $7 \mathrm{yr}$ prior.

Preoperative diagnosis was obtained by digital rectal examination, cysto-urethroscopy, cystourethrogram, and computed tomography scan. We reserve sigmoidoscopy, as a part of the evaluation, for recurrent or radiation-induced RUFs to evaluate the eventual inflammatory proctitis, to exclude a colonic cancer, and to define the extent of radiation injury.

After RUF diagnosis all patients underwent bladder catheterisation along with oral antibiotic therapy and a low residue diet for at least $4 \mathrm{wk}$. Spontaneous fistula closure did not occur in any of the cases.

\subsection{Surgical technique}

\subsubsection{Preoperative preparation and instrumentation}

On the day before surgery, patients were given a clear liquid diet. Bowel clearance was obtained by polyethylene glycol laxative administration the evening before surgery.

In order to perform the procedure we used the Parks' anal retractor, laparoscopic instruments (a $30^{\circ}$ camera, curved scissors, a needle holder, a knot pusher and an aspirator), and a number 15 surgical scalpel blade. To reduce the risk of tissue necrosis we did not use electrocoagulation.

\subsubsection{Patient positioning}

As shown in the Supplementary Video, the operation consisted of an initial cystoscopy followed by the transanal procedure. Initially the patient was placed in the lithotomy position. During cystoscopy a $5 \mathrm{~F}$ catheter was placed within the fistula; it was retracted through the anus, and an $18 \mathrm{~F}$ bladder catheter was introduced. The patient was then placed in a prone jack-knife position.

\subsubsection{Incision of the rectal wall}

After introducing the Parks' anal retractor, the fibrotic margins of the fistula were carefully dissected through a lozenge incision of the rectal wall, performed parallel to the major rectal axis with a number 15 surgical scalpel blade (Fig. 1). Laparoscopic scissors were used to carve the edges of the rectal wall until soft, healthy tissue was reached (Fig. 2). All excised tissue was sent to the pathologist to be analysed.

\subsubsection{Urothelial wall suturing}

Under the healthy flap of the rectal wall the urothelial wall was located and the fistulous tract was sutured, without any additional tissue removal, using interrupted Vicryl 3.0 stitches. In order to close the fistulous tract we passed the first two stitches over and under the $5 \mathrm{~F}$ catheter. The catheter was then removed and we made an additional

Table 1 - Baseline patient characteristics

\begin{tabular}{rlllclr}
\hline Case no. & Age & BMI & pT PCa & Gleason score & Procedure & RUF symptoms appearance (d) \\
\hline 1 & 63 & 28.5 & pT2c & $3+3$ & RRP & 2 \\
2 & 54 & 27 & pT2a & $3+4$ & RRP & 14 \\
3 & 69 & 29 & pT3a & $3+3$ & RRP & 19 \\
4 & 71 & 25.5 & pT2b & $4+3$ & RARP & 16 \\
5 & 48 & 28 & pT3a & $4+3$ & RARP & 24 \\
6 & 57 & 32 & pT2c & $4+5$ & RARP & 9 \\
7 & 69 & 26.5 & pT3a & $4+4$ & RARP & 11 \\
8 & 63 & 30.5 & pT3a & $3+4$ & RARP & 5 \\
9 & 58 & 29 & pT3b & $4+5$ & RARP & 7 \\
10 & 70 & 24 & pT2b & $3+3$ & RARP & 20 \\
11 & 62 & 26 & pT3b & $4+3$ & RARP & 11 \\
\hline 12 & 64 & 29.5 & pT3a & $3+4$ & \\
\hline
\end{tabular}




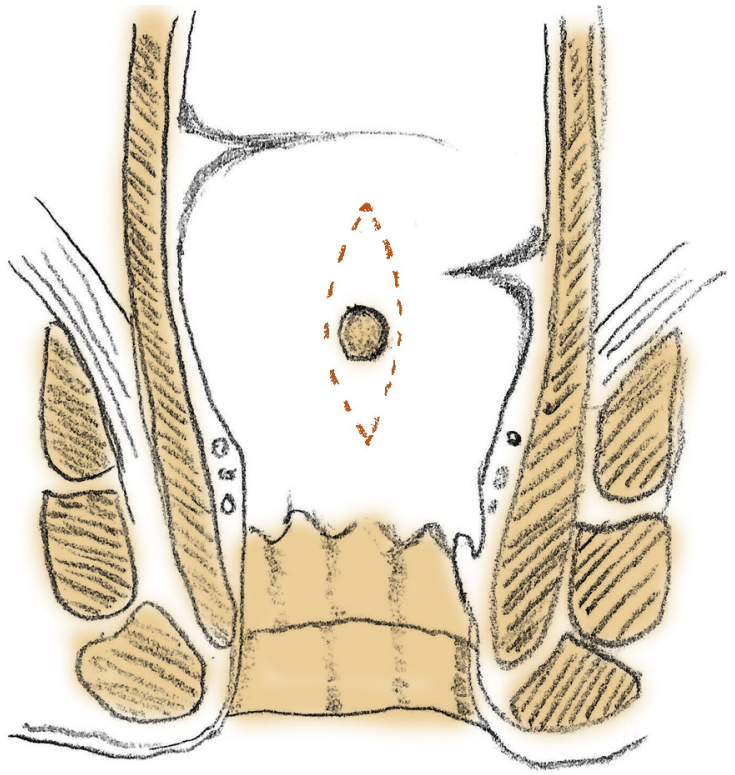

Fig. 1 - Lozenge incision of the rectal wall.

stitch to make the suture watertight. A leakage test of the bladder with $300-\mathrm{ml}$ saline solution was performed.

\subsubsection{Rectal wall suturing}

We then trimmed the incised rectal edges until we obtained a healthy wide soft flap, which, when sewn, slid easily to cover the underlying urothelial sutures. We made deep-bite stitches using 3.0 Vicryl thread and we knotted them. After rectal wall suturing, a second leakage test was performed.

\subsection{Postoperative course}

All patients were admitted to the urological ward. A Visual Analogue Scale questionnaire was completed by all patients on the 1 st postoperative day.

A clear liquid diet was administered after the procedure and continued during Postoperative Day 1, and then a residue-free diet was initiated. Over a week patients gradually advanced to a normal diet. All patients were treated with intravenous ceftriaxone twice a day on the day of surgery, and intramuscular ceftriaxone was administered for $4 \mathrm{~d}$.

All patients were discharged with urinary catheter in situ.

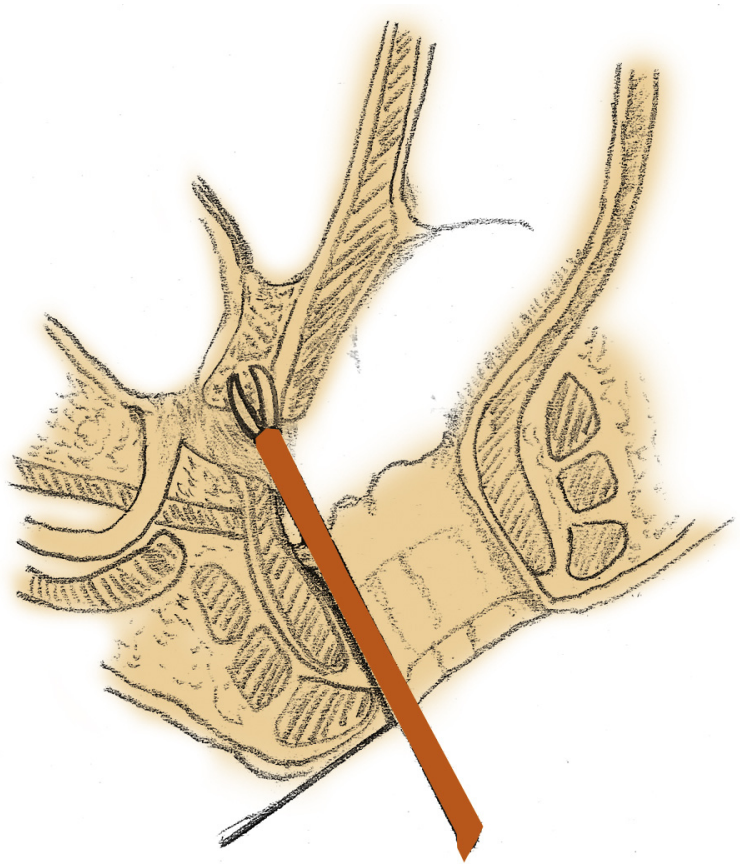

Fig. 2 - Carving the rectal wall's edges in order to reach healthy tissue.

\subsection{Postoperative follow-up}

The bladder catheter was removed $20 \mathrm{~d}$ from surgery following a cystourethrogram examination. Follow-up visits were scheduled at 3 mo and 12 mo postsurgery. A phone interview concerning recurrence and complications was conducted with all patients before data analysis.

We defined successful fistula closure as resolution of clinical symptoms along with radiographic confirmation through cystourethrogram.

\section{Results}

Detailed postoperative results are listed in Table 2. Median follow-up: 21 mo (range, 12-74). Median operative time was $58 \mathrm{~min}$ (range, 50-70). On Postoperative Day 1 patients referred no more than 4 (median: 3 , range, $2-4$ ) on a Visual Analogue Scale $(0=$ no pain, $10=$ unbearable pain $)$. Median hospital stay was $1.5 \mathrm{~d}$ (range, 1-4). Estimated blood loss

Table 2 - Postoperative results

\begin{tabular}{|c|c|c|c|c|c|}
\hline Case no. & Operative time (min) & VAS on Day 1 & Hospital stay (d) & Follow-up (mo) & Time between prostatectomy and MITAR (d) \\
\hline 1 & 60 & 3 & 1 & 74 & 60 \\
\hline 2 & 55 & 3 & 1 & 61 & 69 \\
\hline 3 & 50 & 2 & 2 & 43 & 72 \\
\hline 4 & 60 & 2 & 1 & 31 & 71 \\
\hline 5 & 50 & 3 & 1 & 25 & 76 \\
\hline 6 & 60 & 2 & 4 & 22 & 58 \\
\hline 7 & 70 & 4 & 2 & 20 & 60 \\
\hline 8 & 50 & 3 & 1 & 18 & 78 \\
\hline 9 & 50 & 2 & 2 & 15 & 61 \\
\hline 10 & 60 & 3 & 3 & 13 & 57 \\
\hline 11 & 60 & 3 & 1 & 13 & 73 \\
\hline 12 & 50 & 2 & 2 & 12 & 62 \\
\hline
\end{tabular}


was lower than $50 \mathrm{ml}$ in all cases. Postoperative course was uneventful in all but one patient (8.3\%), who developed cystitis after the procedure and was treated with intravenous antibiotics. The 30-d Clavien complication rate was 8.3\% due to a Grade II complication. No later complications have been observed.

MITAR had been curative in all patients, and no cases of urinary or faecal incontinence occurred secondary to the procedure, or any cases of urethral stenosis. Cancer was not found in any of the specimens. Median time between prostatectomy and MITAR was $66 \mathrm{~d}$ (range, 57-78).

\section{Discussion}

Various surgical strategies for RP can be adopted: retropubic, perineal, laparoscopic, and robotic [9]. Each of these techniques has the risk of accidental rectal wall injury, which is considered a major risk factor for RUF formation [5,7]. During RP the rate of accidental lesions to the anterior rectal wall ranges from $0.12 \%$ to $11 \%$ [5,10]. Intraoperative recognition of these lesions and their treatment reduces the risk of RUFs up to $87 \%$ [5]. Thus, a careful evaluation of anterior rectal wall integrity is advisable during every prostatectomy after the removal of the specimen [5,7,11]. At our institution, after RP we always check for possible iatrogenic rectal damage using the method described by Pisters and Wajsman [12]. If a rectal injury is located, it is repaired by two-layer closure with no tissue interposition.

Among the various approaches to RP, the perineal one is associated with a higher incidence of RUF [7]. At our institution we adopt the retrograde retropubic approach for open RP and the anterograde approach for robotic-assisted RP. Given that the majority of RPs are performed with robotic assistance, eight out of a total of 12 RUFs were secondary to robotic-assisted RP.

In recent years, the great diffusion of radiotherapy and brachytherapy in the treatment of PCa has determined a significant increase of radiation-induced RUFs [13,14]. Indeed, while previous studies reported a radiation-induced fistula rate of $4 \%$, more recently almost $40-50 \%$ of RUFs can be attributable to radiotherapy $[6,15]$. If radiotherapy is administered as neo-adjuvant, during salvage prostatectomy up to $15 \%$ risk of rectal injury is reported because of induced fibrosis in the surrounding tissues [16]. Usually nonradiated RUFs are smaller than radiated or ablative RUFs. Furthermore, fistulas that develop in patients after multimodal PCa treatment are often fibrotic and have hypovascular surrounding tissue [7]. Vanni et al. [15] demonstrated that radiation-induced RUFs can be repaired with muscle flap and buccal mucosal interposition with neither urinary nor faecal diversion, achieving an $84 \%$ success rate. By contrast, Linder et al. [17] reported that 93\% of patients with radiation- or ablation-induced fistulas required permanent urinary diversion and $86 \%$ permanent colostomy. Further data are necessary to assess the best treatment for radiation- or ablation-induced fistula.

RUFs can also appear after RP even if no documented rectal wall damage is found intraoperatively $[3,5]$ and in absence of adjuvant or neo-adjuvant multimodal treatments. These fistulas are usually smaller than radiationinduced RUFs and usually represent the best candidate for MITAR. Eight patients (66.7\%) in our series developed RUF with no intraoperative documented iatrogenic rectal wall damage.

RUF diagnosis is relatively simple. Its symptomatic spectrum consists of rectal passage of urine, pneumaturia, urinary tract infections, and faecaluria $[3,6]$. Faecaluria is a negative prognostic factor because usually it is associated with a large diameter fistula [7]. Faecal diversion is not necessarily routine but should be mandatory in cases of copious faecaluria. As a general rule, when the repair is not tight faecal diversion is advisable as part of the reconstruction [8].

Along with retrograde urethrography and cystoscopy, computed tomography or magnetic resonance imaging allows anatomical and dimensional identification of the fistula. The evaluation of fistula determining factors and symptoms, accompanied by imaging studies, constitutes a crucial step in planning the subsequent approach.

In the absence of faecaluria, for small and nonradiationinduced fistulas a conservative approach may be attempted. This relies on urethral catheterization alone or on urethral catheterization and faecal diversion. Noldus et al. [18] reported that seven of 13 patients (54\%) had spontaneous fistula closure after urethral catheterization for no longer than $3 \mathrm{mo}$. Thomas et al. [7] reported spontaneous fistula closure in three patients managed with urethral catheterization alone after $28 \mathrm{~d}, 29 \mathrm{~d}$, and $100 \mathrm{~d}$, while when performing both urethral catheterization and faecal diversion for fistulas associated with faecaluria or systemic symptoms, literature reports variable percentages of therapeutic success of the conservative treatment, precisely $14 \%$ [19], 33\% [7], and 46\% [20]. Given the lack of uniformity of data, further results are needed to assess the role of conservative approaches in RUF treatment. Indeed, a conservative approach with indwelling bladder catheter should always be attempted. After RUF diagnosis was made, all our patients underwent bladder catheterisation along with oral antibiotic therapy and a residue-free diet. After $4 \mathrm{wk}$, spontaneous fistula healing did not occur in any of the cases.

Concerning surgical options, a review of RUF treatment demonstrated that faecal diversion through ileostomy or, more commonly, colostomy is often performed, and that the perineal approach is the most commonly adopted in preference to the transanal, transabdominal, and transrectal techniques [6]. The Kraske laterosacral technique is not recommended for RUF because of poor urethral exposure [21]. Regardless of the surgical technique adopted, fistula closure can be effective in up to $87.5 \%$ of cases, with a recurrence rate of $12.5 \%$ [6]. Since there is no standardised treatment, the choice of the surgical technique is usually determined by the surgeon's familiarity with a given procedure. The transabdominal approach is the most commonly adopted in cases of radiation or ablationinduced RUF [14,22-24].

The transanal approach is the least commonly used. The Latzko method can accomplish results similar to the other 
techniques [6]. It has the advantage of not involving the division of the anal sphincter. Nevertheless, it is classically criticised because of the limited manoeuvrability of the instruments in the narrow surgical field $[6,25]$. The use of laparoscopic instruments allows the surgeon to overcome this problem. Furthermore, our technique has shown itself to be effective in all patients and can be adopted for treating small fistulas, determining low morbidity and quick recovery.

In literature there is also evidence of minimally invasive transanal approaches for RUF [26-29]. Wilbert et al. [26] reported two cases of RUF managed with transanal endoscopic microsurgery (TEM) along with transurethral fibrin application; in one case colostomy was required because of faecaluria. Quinlan et al. [27] and BochoveOvergaauw et al. [28] exclusively adopted a TEM approach for RUF. Quinlan et al. [27] did not perform faecal diversion, while Bochove-Overgaauw et al. [28] performed a colostomy in both their patients. Atallah et al. [29] used transanal minimally invasive surgery for treating a case of RUF after performing an ileostomy. A rectoscope was used by Wibert et al. [26], Quinlan et al. [27], and Bochove-Overgaauw et al. [28], while Atallah et al. [29] performed the procedure through a SILS port. The fistula was excised with electrocautery by all four study groups [26-29]. In five cases out of six, the transanal minimally invasive procedure resulted effective [26-29]; in one case the procedure, performed after the failure of a gracilo-plasty, failed to resolve the RUF [29]. In TEM and transanal minimally invasive surgery the rectum is distended with carbon dioxide insufflation, whereas we use the Parks' anal retractor to perform MITAR, because it provides good exposure and enough space to work in the surgical field.

Since our 2013 preliminary report of MITAR performed in four patients [30], we have not changed the procedure. Ours is currently the largest case load concerning a minimally invasive transanal approach for treating RUFs.

Some limitations of our study are the small case load, retrospective analysis, and lack of experience for treating fistulas larger than $1.5 \mathrm{~cm}$ or radiation-induced RUFs. We do not exclude the possibility that this procedure is feasible in these circumstances, but faecal diversion may prove necessary. Further results are needed to assess the effectiveness of MITAR under these circumstances.

\section{Conclusions}

MITAR is a safe, feasible, and reproducible procedure performed with laparoscopic instruments on a patient in a prone jack-knife position. Laparoscopic instruments allow us to work in the narrow surgical field and to reduce surgical trauma.

This minimally invasive approach is curative for small fistulas. Since colostomy is not needed, both hospital stay and morbidity are significantly low; moreover, it achieves quick recovery. Careful patient selection is mandatory. Clinical symptoms together with RUF dimension should determine the therapeutic options. Fistulas with a diameter smaller than $1.5 \mathrm{~cm}$ in the absence of sepsis and/or faecaluria can be treated with MITAR.
Further results are needed to assess the effectiveness of this technique in treating larger and/or radiation-induced fistulas.

Author contributions: Giulio Nicita had full access to all the data in the study and takes responsibility for the integrity of the data and the accuracy of the data analysis.

Study concept and design: Nicita.

Acquisition of data: Caroassai Grisanti, Martini, Villari, Li Marzi, Marzocco.

Analysis and interpretation of data: Nicita, Martini, Villari, Caroassai Grisanti.

Drafting of the manuscript: Martini, Nicita.

Critical revision of the manuscript for important intellectual content: Nicita, Villari.

Statistical analysis: None.

Obtaining funding: None.

Administrative, technical, or material support: None.

Supervision: Nicita.

Other (video editing and images): Martini, Marzocco.

Financial disclosures: Giulio Nicita certifies that all conflicts of interest, including specific financial interests and relationships and affiliations relevant to the subject matter or materials discussed in the manuscript (eg, employment/affiliation, grants or funding, consultancies, honoraria, stock ownership or options, expert testimony, royalties, or patents filed, received, or pending), are the following: None.

Funding/Support and role of the sponsor: None.

Acknowledgments: Thanks to Professor Brenda Porster and Professor Gail Solberg for the linguistic revision.

\section{Appendix A. Supplementary data}

Supplementary data associated with this article can be found, in the online version, at http://dx.doi.org/10.1016/j. eururo.2016.06.006.

\section{References}

[1] Elliott SP, McAninch JW, Chi T, Doyle SM, Master VS. Management of severe urethral complications of prostate cancer therapy. J Urol 2006;176:2508-13.

[2] Ghoniem G, Elmissiry M, Weiss E, Langford C, Abdelwahab H, Wexner S. Transperineal repair of complex rectourethral fistula using gracilis muscle flap interposition-can urinary and bowel functions be preserved? J Urol 2008;179:1882-6.

[3] Kitamura H, Tsukamoto T. Rectourinary fistula after radical prostatectomy: review of the literature for incidence, aetiology, and management. Prostate Cancer 2011;2011:629105.

[4] Novara G, Ficarra V, Rosen RC, et al. Systematic review and metaanalysis of perioperative outcomes and complications after robotassisted radical prostatectomy. Eur Urol 2012;62:431-52.

[5] Roberts WB, Tseng K, Walsh PC, Han M. Critical appraisal of management of rectal injury during radical prostatectomy. Urology 2010;76:1088-91.

[6] Hechenbleikner EM, Buckley JC, Wick EC. Acquired rectourethral fistulas in adults: a systematic review of surgical repair techniques and outcomes. Dis Colon Rectum 2013;56:374-83.

[7] Thomas C, Jones J, Jaeger W, Hampel C, Thueroff JW, Gillitzer R. Incidence, clinical symptoms and management of rectourethral fistulas after radical prostatectomy. J Urol 2010;183:608-12. 
[8] Zinman L. The management of the complex recto-urethral fistula. BJU Int 2004;94:1212-3.

[9] Heidenreich A, Bastian PJ, Bellmunt J, et al. EAU guidelines on prostate cancer. Part II: Treatment of advanced, relapsing, and castration-resistant prostate cancer. Eur Urol 2014;65:467-79.

[10] Lassen PM, Kearse Jr WS. Rectal injuries during radical perineal prostatectomy. Urology 1995;45:266-9.

[11] Katz R, Borkowski T, Hoznek A, Salomon L, De La Taille A, Abbou CC. Operative management of rectal Injuries during laparoscopic radical prostatectomy. Urology 2003;62:310-3.

[12] Pisters LL, Wajsman Z. A simple test for the detection of intraoperative rectal injury in major urological pelvic surgery. J Urol 1992; $148: 354$.

[13] Theodorescu D, Gillenwater JY, Koutrouvelis PG. Prostatourethralrectal fistula after prostate brachytherapy. Cancer 2000;89:2085-91.

[14] Larson DW, Chrouser K, Young-Fadok T, Nelson H. Rectal complications after modern radiation for prostate cancer: a colorectal surgical challenge. J Gastrointest Surg 2005;9:461-6.

[15] Vanni AJ, Buckley JC, Zinman LN. Management of surgical and radiation induced rectourethral fistulas with an interposition muscle flap and selective buccal mucosal onlay graft. J Urol 2010;184: $2400-4$.

[16] Kimura M, Mouraviev V, Tsivian M, Mayes JM, Satoh T, Polascik TJ. Current salvage methods for recurrent prostate cancer after failure of primary radiotherapy. BJU Int 2010;105:191-201.

[17] Linder BJ, Umbreit EC, Larson D, Dozois EJ, Thapa P, Elliott DS. Effect of prior radiotherapy and ablative therapy on surgical outcomes for the treatment of rectourethral fistulas. J Urol 2013;190:1287-91.

[18] Noldus S, Fernandez H, Huland. Rectourinary fistula repair using the Latzko technique. J Urol 1999;161:1518-20.

[19] Nyam DC, Pemberton JH. Management of Iatrogenic rectourethral fistula. Dis Colon Rectum 1999;42:994-7.
[20] al Ali M, Kashmoula D, Saoud IJ. Experience with 30 posttraumatic rectourethral fistula: presentation of posterior trans-sphinteric anterior rectal wall advancement. J Urol 1997;158:421-4.

[21] Dafnis G, Wang YH, Borck L. Transsphincteric repair of rectourethral fistulae following laparoscopic radical prostatectomy. Int J Urol 2004;11:1047-9.

[22] Lane BR, Stein DE, Remzi FH, Strong SA, Fazio VW, Angermeier KW. Management of radiotherapy induced rectourethral fistula. J Urol 2006;175:1382-7.

[23] Moreira Jr SG, Seigne JD, Ordorica RC, Marcet J, Pow-Sang JM, Lockhart JL. Devastating complications after brachytherapy in the treatment of prostate adenocarcinoma. BJU Int 2004;93:31-5.

[24] Netsch C, Bach T, Gross E, Gross AJ. Rectourethral fistula after highintensity focused ultrasound therapy for prostate cancer and its surgical management. Urology 2011;77:999-1004.

[25] Gupta G, Kumar S, Kekre NS, Gopalakrishnan G. Surgical management of rectourethral fistula. Urology 2008;71:267-71.

[26] Wilbert DM, Buess G, Bichler KH. Combined endoscopic closure of rectourethral fistula. J Urol 1996;155:256-8.

[27] Quinlan M, Cahill R, Keane F, Grainger R, Butler M. Transanal endoscopic microsurgical repair of iatrogenic recto-urethral fistula. Surgeon 2005;3:416-7.

[28] Bochove-Overgaauw DM, Beerlage HP, Bosscha K, Gelderman WA. Transanal endoscopic microsurgery for correction of rectourethral fistulae. J Endourol 2006;20:1087-90.

[29] Atallah SB, deBeche-Adams TC, Larach S. Transanal minimally invasive surgery for repair of rectourethral fistula. Dis Colon Rectum 2014;57:899.

[30] Nicita G, Li Marzi V, Mencarini M, Cocci A, Villari D, Marzocco M. Mini-invasive transrectal repair of recto-urinary fistula after radical prostatectomy. J Urol 2013;4:e372-3. 